\title{
A Product Life Cycle Ontology for Additive Manufacturing
}

\author{
Munira Mohd Ali ${ }^{\mathrm{a}, *}$, Rahul Rai ${ }^{\mathrm{a}}$, J. Neil Otte ${ }^{\mathrm{b}}$, Barry Smith ${ }^{\mathrm{b}}$ \\ ${ }^{a}$ Department of Mechanical and Aerospace Engineering, State University of New York at \\ Buffalo, Buffalo, NY, USA \\ ${ }^{b}$ National Center for Ontological Research, State University of New York at Buffalo, Buffalo, \\ $N Y, U S A$
}

\begin{abstract}
The manufacturing industry is evolving rapidly, becoming more complex, more interconnected, and more geographically distributed. Competitive pressure and diversity of consumer demand are driving manufacturing companies to rely more and more on improved knowledge management practices. As a result, multiple software systems are being created to support the integration of data across the product life cycle. Unfortunately, these systems manifest a low degree of interoperability, and this creates problems, for instance when different enterprises or different branches of an enterprise interact. Common ontologies (consensusbased controlled vocabularies) have proved themselves in various domains as a valuable tool for solving such problems. In this paper, we present a consensusbased Additive Manufacturing Ontology (AMO) and illustrate its application in promoting re-usability in the field of dentistry product manufacturing.

Keywords: additive manufacturing ontology, manufacturing process ontology, ontology engineering, product life cycle, dentistry product manufacturing
\end{abstract}

\footnotetext{
Preprint version of article published in Journal of Computers in Industry, Volume 105, February 2019, Pages 191-203. (https://doi.org/10.1016/j.compind.2018.12.007) .

is Only to be used for personal use, copyright belongs to the authors and Elsevier Science.

${ }^{*}$ Corresponding author

Email addresses: munirabi@buffalo.edu (Munira Mohd Ali), rahulrai@buffalo.edu (Rahul Rai), jeffotte@buffalo.edu (J. Neil Otte), phismith@buffalo.edu (Barry Smith)
}

Preprint version of paper published in Computers in Industry, 105 (2019), 191-203 https://doi.org/10.1016/j.compind.2018.12.007 


\section{Introduction}

The economic model of the manufacturing industry is increasingly based on the modularization of industrial processes and digitally mediated collaboration between modules both internally, within the enterprise, and externally, through

5 subcontracting and off-shoring. The sharing of information is crucial for facilitating such collaboration across all phases in the life of a product from development and design, through production and sale, to use and disposal [1, 2, 3]. As industrial processes come to be further modularized and distributed throughout the entirety of the manufacturing pipeline, more powerful and more intelligent software solutions are required to support the different components and phases of the product life cycle (PLC).

The Economist Intelligence Unit 44 reports that the need for knowledge representation of manufacturing processes is increasing exponentially as technology expedites the rapid exchange of information. In information science, an ontology is a controlled vocabulary implemented in a semantic or knowledge representation language such as the Web Ontology Language (OWL). Ontologies have been successfully used, for example, in military domains and biomedicine. In the design and manufacturing domains, in contrast, the use of ontologies has not lived up to initial expectations, due not least to a lack of coordination among industrial enterprises. The focus of this paper is to present the Additive Manufacturing Ontology (AMO), an ontology designed to represent the Additive Manufacturing (AM) Product Life Cycle.

AMO is a modular ontology that employs Basic Formal Ontology as its top level, while also drawing from the Common Core Ontologies and three other ontologies from the Coordinated Holistic Alignment of Manufacturing Process Ontologies that represent manufacturing processes: the Manufacturing 
Process Ontology, the Design Ontology, and the Testing Process Ontology. As an illustrative example, the use of AMO is illustrated in the dentistry manufacturing domain.

\section{Related Work}

\subsection{Ontology Development in the Manufacturing Domain}

Many manufacturing ontologies have been developed in recent years. For example, Lemaignan et al. [5] developed the Manufacturings Semantics Ontology (MASON), which employs three top-level classes of entities, operations, and resources. Entities in MASON comprise a broad class including geometric entities (for example, shape), raw materials, and costs. Operations class attempts to cover all processes involved in manufacturing, and resources attempt to represent tools, human resources, and geographic resources. MASON was developed as an upper-level ontology to accomplish two goals:

1. Developing an architecture and tools for automatic cost estimation, and

2. Linking a high-level ontology written in OWL with a multi-agent framework for manufacturing simulation.

Unfortunately, MASON's tripartite division of classes into entities, operations, and resources are lack of classificatory coherence. Entity in MASON, for instance, 45 is introduced as comprising the common helper concepts used to specify a product. However, entity as defined in the OWL Web Language Guide [6] and OWL2 Web Ontology Language Structural Specification [7] does not limit the definition in specific concept of specifying product only but also include classes, datatypes, object properties, data properties, annotation properties, and named individuals are entities.

Kjellberg et al. [8] introduces the Machine-Tool Model (MTM) as an ontology focusing on the machine tool as a central part of a manufacturing system as 
well as on the way machine tool information is used throughout the design and operation of such systems. Process planning, for instance, requires information on the functional properties of machine tools, such as the ability to perform different types of machining operations.

Both MASON and the MTM were developed from scratch, each in its ad hoc way, and they do not use a common upper-level ontology nor do they reuse the content of other domain ontologies. In this way, they re-create the very lack of interoperability that they were designed to address, but now this lack occurs between ontologies rather than between data systems. Even though MASON was intentionally developed as an upper-level ontology to represent manufacturing information, the entities in MASON are identifiable and concrete to represent manufacturing as the specialized domain of interest instead of being an ontology

65 that is domain neutral. According to Musen [9], upper-level ontology is defined as an ontology at a sufficiently high level of abstraction such that it does not refer to identifiable, concrete entities in the domain of interest.

MASON's contribution as an upper-level ontology is undeniably has contributed to the development of other ontologies in manufacturing domain. For instance, Ramos [10] introduces the Machine Ontology (MO), which elaborates the representation of machines in terms of the market, material, and operation features. The resultant redundancy between MASON, MTM, and MO led Ramos et al. 11] to present a method for integrating ontology reuse with ontology validation, and they applied this method to the three ontologies in question, using Protêgê-Prompt to find common content and overlapping terms between them. The Machine of a Process Ontology (MOP) was developed as a result of this work with the goal of facilitating the buying and selling of industrial machinery; it employs MASON as its reference ontology while drawing in relevant classes and relations from MTM and MO. The CDM-Core Ontology, presented by Mazzola 
et al. 12] is another ontology that was developed by reusing MASON as one of the upper-level ontology. CDM-Core Ontology includes both the general manufacturing domain applicability and the specific project use cases that can be a guidance for developing other specific applications in manufacturing domain.

Another ontology, the Manufacturing Service Description Language (MSDL), was introduced by Ameri et al. [13, who employ a methodology relying on the incremental enhancement of an initial set of definitions constructed on the basis of a formal ontology. MSDL is an upper-level ontology that supports the semantic framework for representing conventional manufacturing processes outlined in Kjellberg et al. [8]. The original purpose of MSDL was to serve as the ontology in an agent-based framework for supply chain deployment; for this reason, it employs an analysis of manufacturing capabilities across several levels: the supplier, shop, machine, device, and process.

Mesmer and Olewnik 14 proposes a Part-Focused Manufacturing Process Ontology (PMPO) designed around the idea that a classification of manufacturing processes can be developed on the basis of an account of the desired features and attributes of the products they will be used to manufacture. The ontology thus develops a representation of the qualities used in specifying product requirements, including material composition, cost, shape, size, the surface finish of the product, thickness, and so forth. Users can describe the features and attributes based on the qualities defined in $\mathrm{PMPO}$, and select appropriate manufacturing processes according to the information provided.

Most ontologies designed for the manufacturing domain thus far have been put together with a focus narrowly directed to some specific sub-domain of manufacturing engineering and with little attention to interoperability with other ontologies in related domains. Among the ontologies discussed, MOP and PMPO stand out because they build on prior work. PMPO is especially 
interesting in that it utilizes not only Basic Formal Ontology but also MSDL, the Ontology for Biomedical Investigations (OBI), and the Common Semantic Model Ontology (COSMO). It is thus, at least to some degree, able to achieve interoperability among data systems deriving from external sources. On the other hand, PMPO is small in scale and has been designed only for traditional machining and molding processes thus it cannot be applied to more modern manufacturing processes such as additive manufacturing.

Ideally, a representation of the manufacturing domain should deal with commonly collected product-related information. Moving forward, we hold that an ontological representation of products and the PLC is a prerequisite for integrating data across systems in the manufacturing domain. Therefore, developing an ontology with a focus on AM products - their qualities, functions, the production, use, and end-of-life is the main objective of this paper. However, our ontology is intended to form part of a larger suite of modular ontologies within the framework of the Industrial Ontologies Foundry (IOF) initiative, and it will accordingly be modified in tandem with IOF development. IOF is an initiative that was proposed to promote interoperability of high quality and non-redundant ontologies in industrial domains or manufacturing specializations [15].

\subsection{Manufacturing Processes and the Product Life Cycle (PLC)}

As customer demands diversify, the complexity of products and product repertoires increases, and this gives rise to demand for increasingly innovative manufacturing processes [16]. Understanding the nature of such processes and creating computational systems that can understand and reason about them is crucial, and this means understanding and reasoning across the entire product life cycle (PLC) in the Product Life Cycle Management (PLM). Moreover, Product, Process and Resource (PPR) are the key elements of engineering 
domain in any manufacturing industry [17. The information about PPR that is structured in PLM systems requires explicit mapping among the PPR for a complex decision purpose. Therefore, an ontology that provides common vocabularies in representing knowledge could facilitate the full potential of the PLM by supporting information exchange between the PPR in different phases in PLC 17, 18.

1. Marketing Product Life Cycle (M-PLC) models that focus primarily upon marketing needs and conceptions;

2. Engineering Product Life Cycle (E-PLC) models that integrate design and manufacturing with marketing needs and conceptions.

Figure 1 represents the successive phases of the PLC taken as the basis of ontology development in many recent works, including Young et al. [3, Chen et al. [16, Borsato [18, Matsokis and Kiritsis [20, Chungoora et al. [21, Usman et al. 22], Urwin [23, Urwin et al. 24], and others.

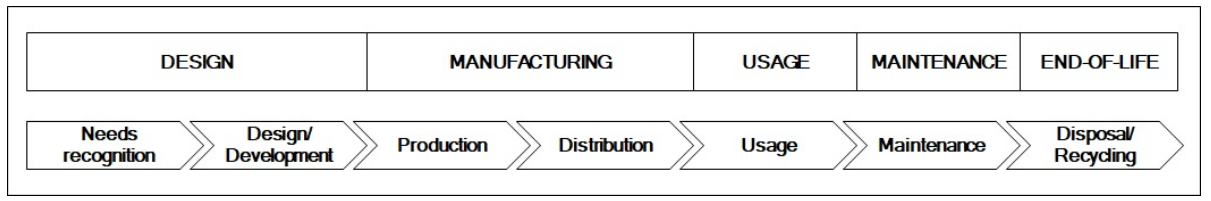

Figure 1: Phases in the PLC.

Table 1, from Chen et al. [16], extends this representation to create a more granular perspective. Here, the PLC is depicted as consisting of seven stages each with a number of sub-stages.

Chen et al. [16, Borsato [18, Matsokis and Kiritsis [20, Chungoora et al. [21, Usman et al. 22, Urwin [23] and Urwin et al. 24] have demonstrated good concept of information integration and sharing between the design and 155 manufacturing phases in PLC through ontology. Borsato [18] for instance, 
Table 1: Seven stages of the PLC presented by Chen et al. 16]

\begin{tabular}{|c|c|}
\hline Stages & Sub-stages \\
\hline Product design & $\begin{array}{l}\text { Requirement analysis, Conceptual design, Prelimi- } \\
\text { nary design, Detail design }\end{array}$ \\
\hline Process development & $\begin{array}{l}\text { Part description, Generative process planning, Vari- } \\
\text { ant process planning }\end{array}$ \\
\hline Product manufacturing & $\begin{array}{l}\text { Equipment layout, Production management, Qual- } \\
\text { ity control }\end{array}$ \\
\hline Sales & $\begin{array}{l}\text { Chance analysis, Target market choice, Sell combi- } \\
\text { nation development }\end{array}$ \\
\hline Product in use & $\begin{array}{l}\text { Operation instructions establishment, Product in- } \\
\text { stallation and execution }\end{array}$ \\
\hline Post sell service & $\begin{array}{l}\text { User problem reaction, Problem identification, Ser- } \\
\text { vice support }\end{array}$ \\
\hline Product retirement & Decomposition, Recycling \\
\hline
\end{tabular}

believes that ontology could bridge the gap between manufacturing and PLC. Chungoora et al. 21] presents the Interoperable Manufacturing Knowledge System (IMKS) model-driven concept that was built on the ideas of extensible core ontologies of manufacturing. Usman et al. 22] forms the Manufacturing Core Concepts Ontology (MCCO) by identifying core set of concepts formalized in the upper-level ontology that serve as foundation ontology to provide the first stage of a common understanding before developing the domain specific concepts of design and production.

In what follows, we will adopt this granular perspective in conceiving of the PLC as having a scope that includes processes of design and development, manufacturing, usage, maintenance and disposal, as well as the information, materials, qualities, and functions that participate in these processes.

In particular, we take into account also the following key areas of interest regarding the PLC identified by Young et al. [3]:

- Information regarding products including product geometry,

- Potential supply chain capability, 
- Knowledge of what has been done in the past, and

- Potential legislation, catalog data, and standards that affect decision making.

175 mechanical properties for metal-based AM of Ti-6-6Al-4V. Liang [30 develops the AM-OntoProc ontology that promote the modeling and reutilization of 
knowledge towards the AM process planning where AM process is supposed to begin from the utilization of CAD software during the design stage until the final AM prototype is developed.

Finally, there is recent work by Hagedorn et al. 31, which uses BFO as the platform for an AM ontology called Innovative Capabilities of Additive Manufacturing (ICAM). ICAM also reuses the BFO-conformant ontology - the Information Artifact Ontology (IAO) - to provide the higher-level representation of information-related types that serves as its backbone. The information in ICAM covers basic product attributes from the NIST Core Product Model $(\mathrm{CPM})$ relating to materials, geometry and designed function as well as types of manufacturing processes and services taken over from MSDL. It also incorporates the SAMPro model of AM and a set of formal description of parts and features from Functional Basis Ontology (FBO). ICAM is thus able to provide extensive coverage of the AM domain and since our version of AMO also reuses the BFOconformant ontology - the Common Core Ontologies (CCO), we will be looking forward for the opportunity to develop future versions of AMO to be consistent with the ICAM content that focus on the application of AM. The CCO is a suite of ontologies that was released to the public recently that adds general contents to the $\mathrm{BFO}$ structure and at the same time are also common to many domain of interest, especially to manufacturing engineering domain. We feel that Information Entity Ontology (IEO) that is part of CCO seems to be able to represent information-related types to manufacturing domain in a more accurate way than IAO. However, there are still works to be done in making the IEO and IAO to be compatible to each other. 


\section{Approach for Ontology Development}

Although there are is no standard methodology for developing ontologies, 225 Natalya F and Deborah L [32] outlined a simple knowledge-engineering methodology in developing ontology which was followed as a guideline in our work. The methodology include: determining ontology domain and scope, considering ontology reuse, enumerating important terms, defining classes and class hierarchies, defining class properties, defining values for properties, and creating instances of parts of the ontologies overlapped with each other. Since our work is part of the Coordinated Holistic Alignment of Manufacturing Processes (CHAMP) project founded by Digital Manufacturing and Design Institute (DMDII) which focuses on constructing an efficient scheme to manage discordant manufacturing data 

despite of the selection of BFO due to the CHAMP project requirement, BFO's well-documented guidelines and training material, it's extensive use in in hundreds of projects in biomedical and military domains, and increasingly being adopted in industry as a top-level framework are another factors that contribute to the

A fragment of the BFO class hierarchy is provided in Figure 2.

An AM process is a process that involves certain sorts of material entities as its participants. A Portion of Material is a subclass of material entity, and

\footnotetext{
${ }^{1}$ See https://github.com/BF0-ontology/BFO/blob/master/bfo.owl

${ }^{2}$ See https://github.com/CommonCoreOntology/CommonCoreOntologies
} 


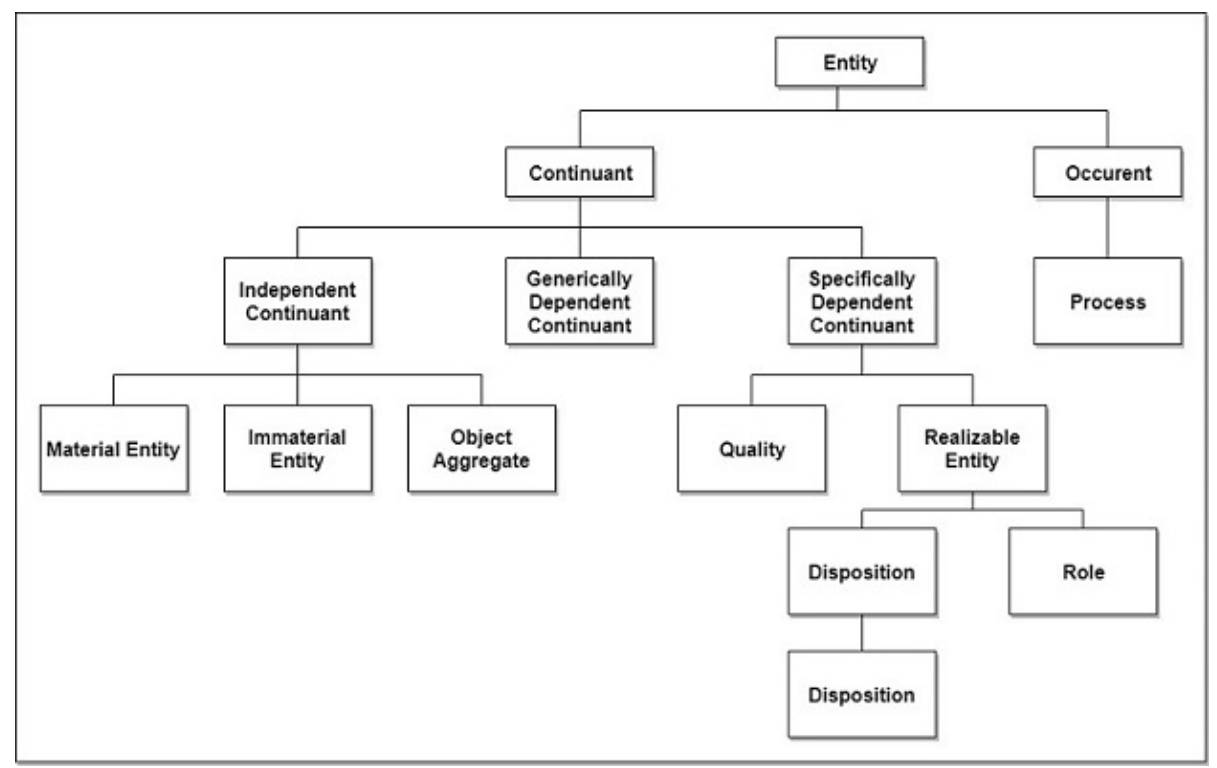

Figure 2: A fragment of the BFO class hierarchy [39].

instances of Portion of Material are the inputs for instances of AM process.

Both processes and material entities are distinct from the third type of entity in BFO comprising generically dependent continuants. These are, roughly, patterns that can be exactly copied - they are entities that depend on the existence of at least one bearer at any time during which they exist, but not on any particular bearer. The most important subclass of generically dependent continuant is Information Content Entity, whose instances stand in a relation of aboutness to some entity [40. Importantly, for our purposes, this class includes instances of reports, sentences, and data values that are about the processes and materials of a manufacturing process. Such information is not itself material, nor is it a process, though it may participate in processes [34].

The Common Core Ontologies (CCO) form a set of conservative extensions of $\mathrm{BFO}$ with the goal of representing the mid-level entities involving agents, artifacts, actions, and measurements [1]. The ontologies in the CCO include: 
- Agent Ontology, representing agents, especially persons and organizations,

290

- Artifact Ontology, representing deliberately created material entities along with their models, specifications, and functions.

- Currency Unit Ontology, representing currencies in different countries.

- Extended Relation Ontology, representing relations (i.e. object properties) Every class in $\mathrm{CCO}$ is the subclass of some class in $\mathrm{BFO}$, and general relations used in BFO are also adopted by the CCO. 


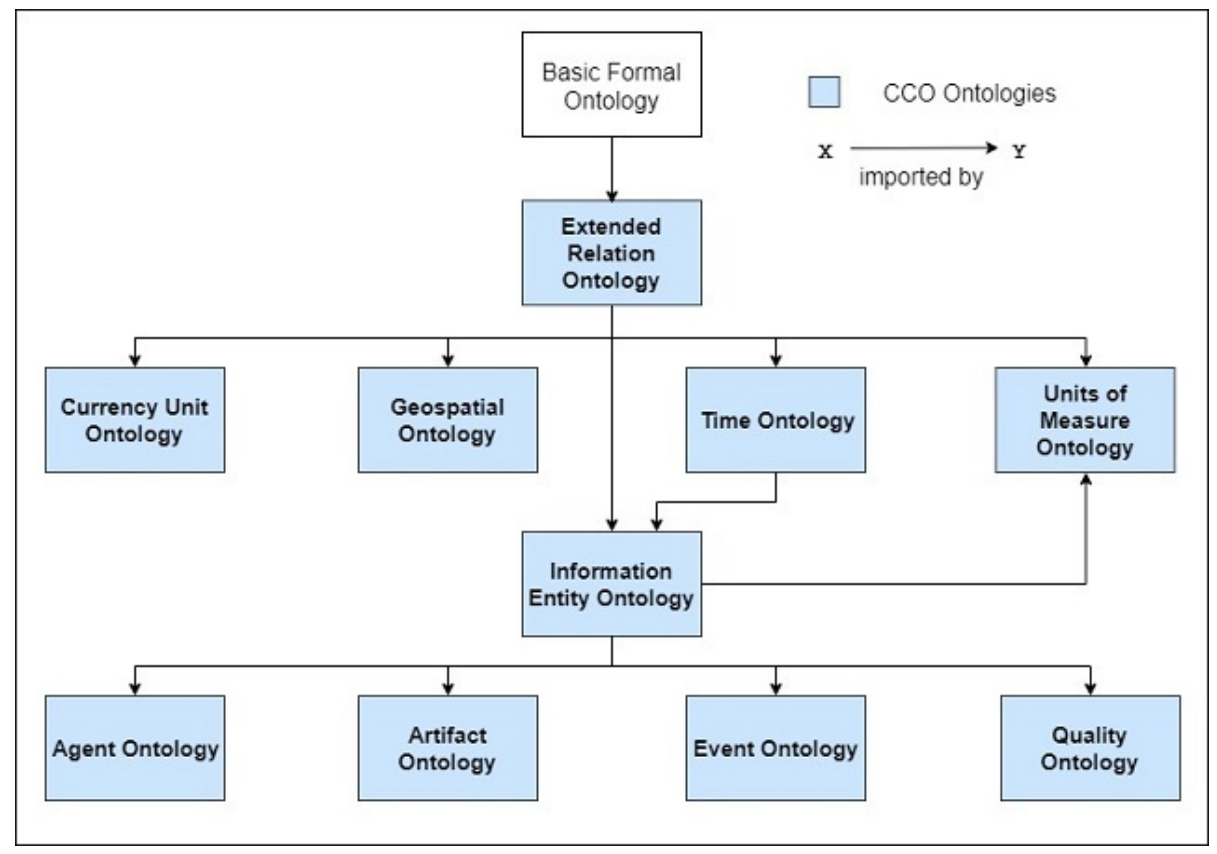

Figure 3: The Common Core Ontology import structure [37.

Although CCO served as the mid-level ontology that reduce the generality of BFO, having direct children from CCOs' classes to represent the specific domain in AM are not sufficient. There is a need of another level of ontology after the $\mathrm{CCO}$ so that it can increase the granularity of domain before moving towards the concrete entities that represent AM. The Coordinated Holistic Alignment of Manufacturing Processes (CHAMP) is a project funded by the Digital Manufacturing and Design Innovation Institute (DMDII). The CHAMP 320 project has developed a suite of ontologies whose objective is to aid industrial organizations in overcoming the problem of data heterogeneity. The CHAMP ${ }^{3}$ ontologies are an extension of $\mathrm{CCO}$ representing the mid-level classes relating to the design, manufacturing, use, and maintenance phases of the PLC. These ontologies include:

\footnotetext{
${ }^{3}$ See https://github.com/NCOR-US/CHAMP
} 
Figure 4 shows the CHAMP ontologies and their import structure.

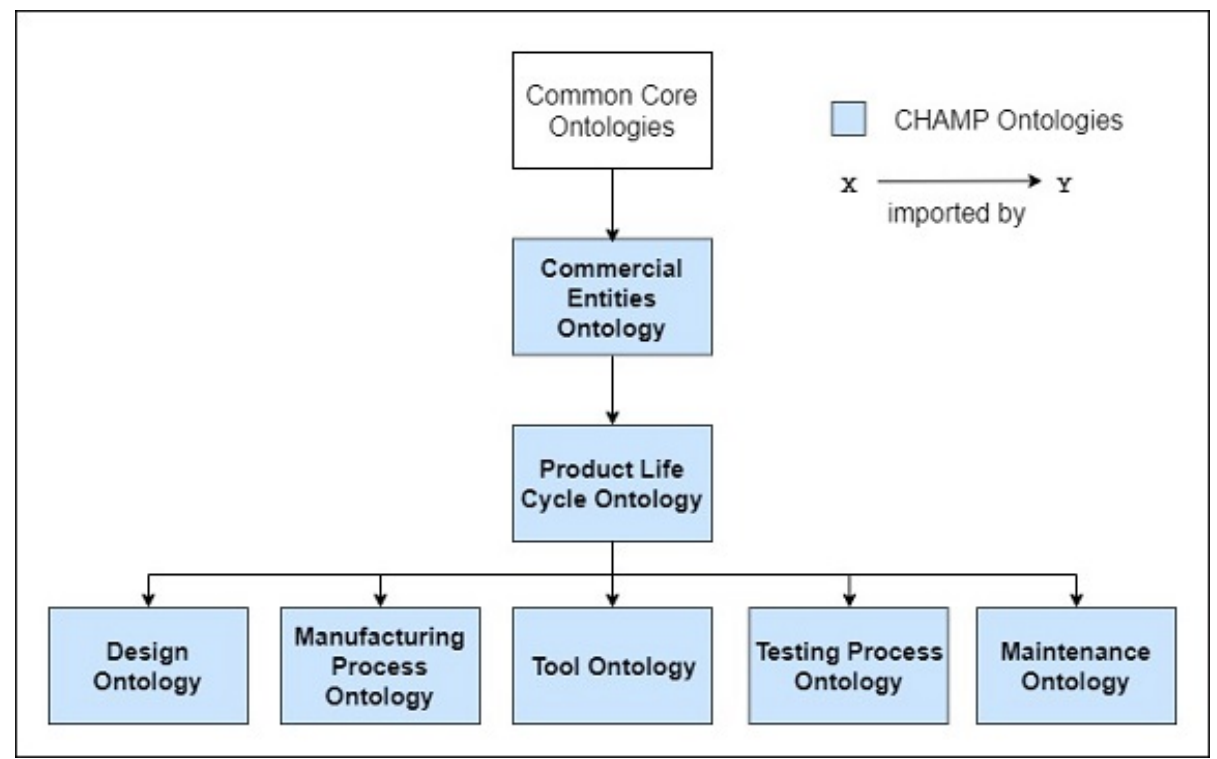

Figure 4: The CHAMP Ontologies

Ideally, we planned to extend the whole set of CHAMP ontologies for the development of AMO. However, since the CHAMP ontologies are still in initial implementation, we decided to import only a fragment of the CHAMP ontologies that are related to the AM processes. We imported manually some of the 
classes from the Design Ontology, Manufacturing Process Ontology, and Testing Process Ontology without changing its' class structures and URI from its' original ontologies. Figure 5 shows the framework for the AMO development.

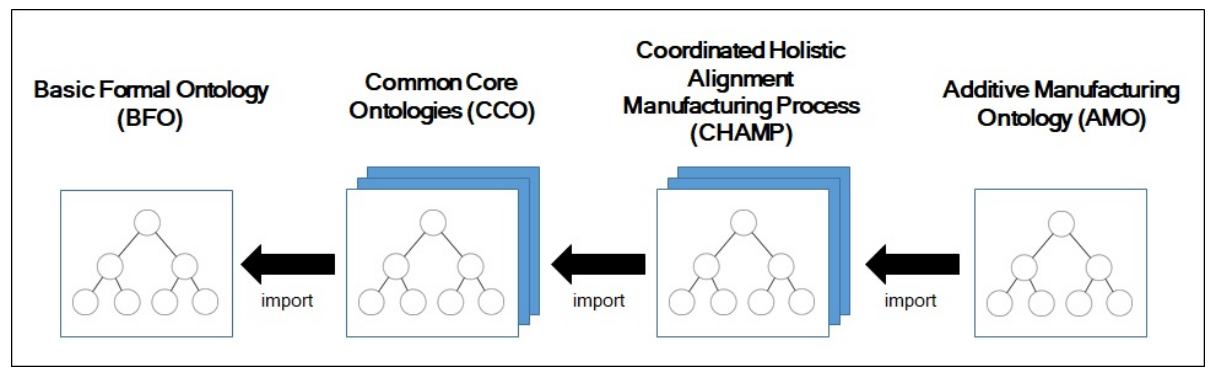

Figure 5: An overview of AMO development.

\section{The Additive Manufacturing Ontology (AMO)}

Existing classes from BFO, CCO, and CHAMP are imported into AMO and new classes are added in a process of downward population. This ensures that AMO utilizes commonly used terms and definitions and thereby increases the chances that AMO will itself be re-used and integrated with other ontologies.

There is a canonical order to processes that occur within AM, and that the processes represented in the AMO were selected in order to account for this canonical representation. From a mechanical perspective, AM often uses numerically controlled (NC) machines that are integrated with CAD and process planning software. The canonical AM process flow consists of six steps [42]:

1. 3D CAD model generation;

2. Conversion of the CAD Model into AM machine acceptable format (STL file);

3. Setting the process parameters; 
5. Support removal; and

6. Post-processing.

The class process is defined in $\mathrm{BFO}$ as an occurrent entity that exists in time by occurring or happening, has temporal parts, and always depends on some (at least one) material entity 34]. Each of the steps listed by Yang et al. 42 corresponds to the instantiation of a certain type of process entity. Process entities in AMO include:

1. Design Ontology: ActOfDescribingClientNeed

2. Design Ontology: ActOfAnalysisOfClientNeed

3. AMO: ActOfCADModelDevelopment

4. AMO: ActOfDataTransformation

5. Design Ontology: ActOfManufacturingRequirementIdentification

6. Manufacturing Process Ontology: ActOfAdditiveManufacturing

7. AMO: ActOfSupportRemoval

8. AMO: ActOfPostProcessing

As can be seen, some of these classes are imported from the Design Ontology and the Manufacturing Process Ontology. Figure 6 shows the taxonomy of the process classes in AMO. IntentionalAct as can be seen from the figure is the subclass of Act class where both Act and IntentionalAct are the CCO classes, extension of Process class in BFO. The definition of both class as follows:

- Act is a process in which at least one agent plays a causative role.

- IntentionalAct is an Act in which at least one agent plays a causative role and which is prescribed by some Directive Information Content Entity held by at least one of the Agents. 


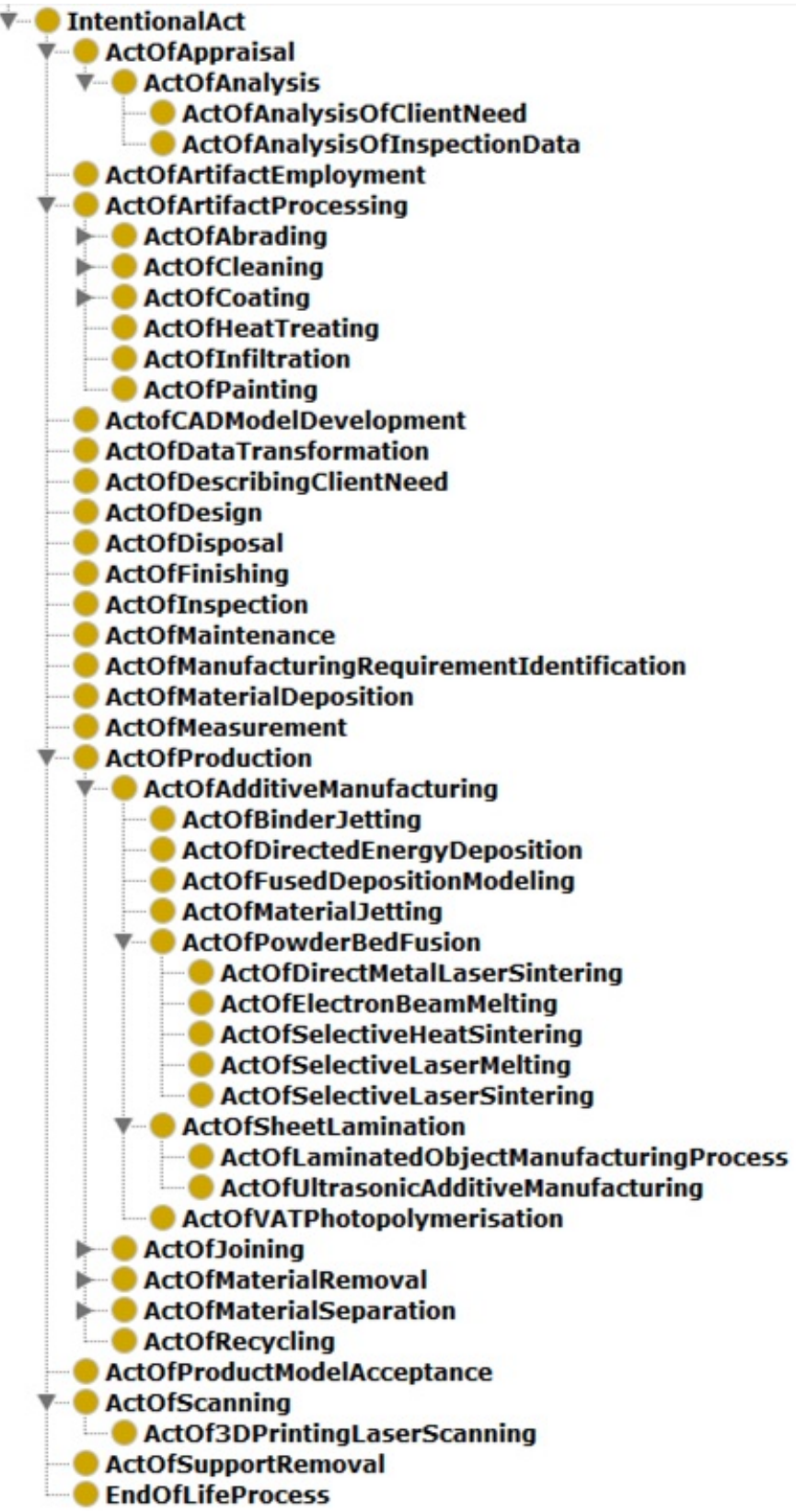

Figure 6: Process classes in AMO. 
process. However, it is not right to put that process class as the subclass of the main process since this process part relations only hold in some cases and not all the time. Figure 7 shows the example of some ActOfSupportRemoval that 385 has_process_part an ActOfMaterialRemoval and an ActOfPostProcessing that has an ActOfAbrading and an ActOfJoining as process parts.

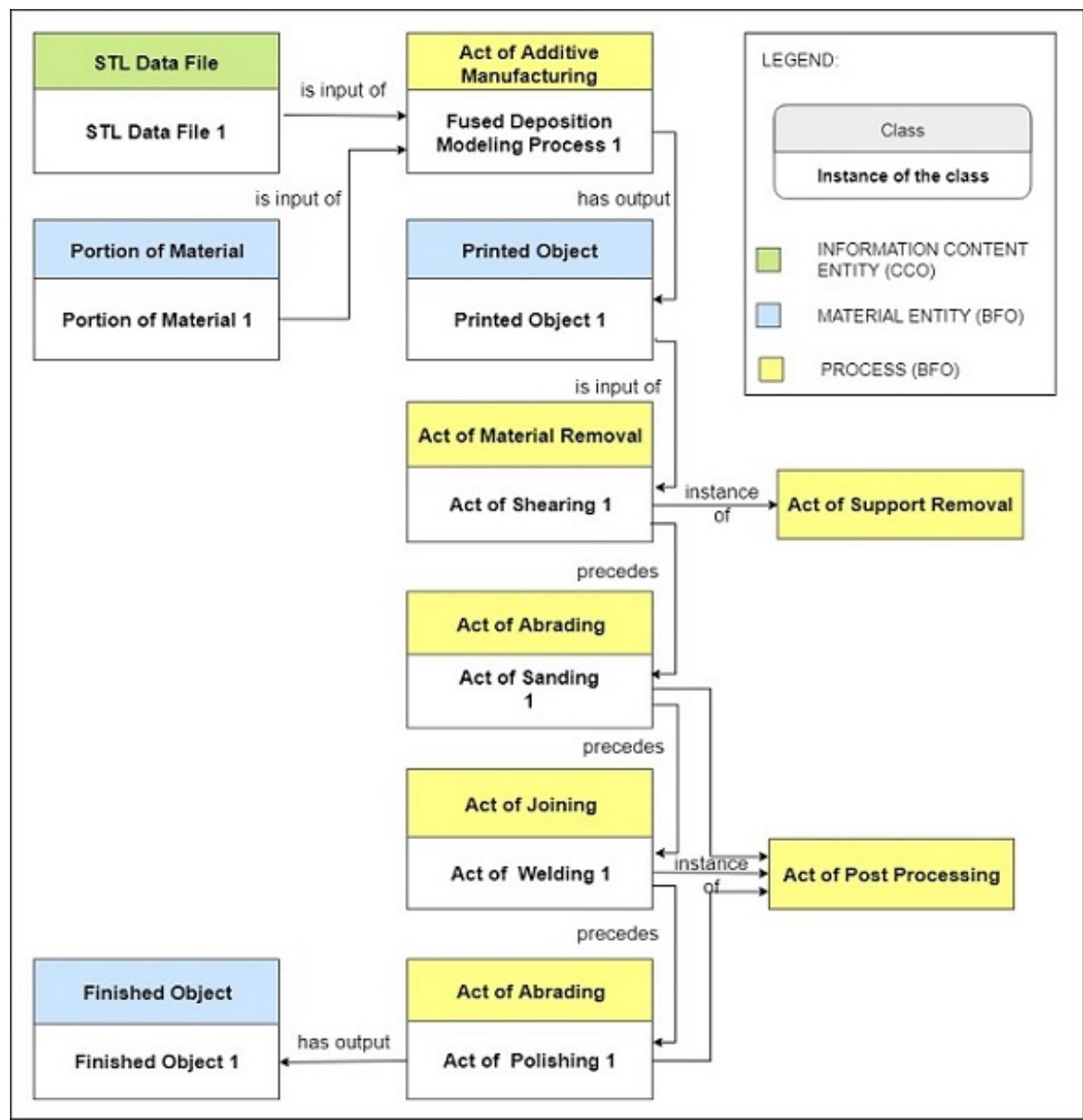

Figure 7: Example for process parts of Act of Support Removal and Act of Post Processing. 


\subsection{Material Entity}

BFO defines a material entity as an independent continuant that has some portion of matter as part [34. Three types of material entity are recognized by BFO: object, fiat object part, and object aggregate [34]. BFO does not assert that all material entities fall under one or other of these headings. Thus, portions of liquid, gas, and plasma are classified (in the current version of BFO) as immediate descendants of material entity.

Resources involved in the AM process can all be classified as material entities in the AMO. These resources are:

- Portion of Material

- 3D Printing Machine

- Printed Object

- Finished Object

Portion of Material in AMO is a direct child of material entity because it may describe those material entities that are not object aggregates. Material entity classes in AMO are shown in Figure 8. Meanwhile, 3DPrintingMachine, PrintedObject, and FinishedObject are types of object in BFO [43. PrintedObject and FinishedObject are defined classes with definitions as follows:

405

\subsection{Information Content Entity}

An Information Content Entity as defined by the CCO is "a Generically 410 Dependent Continuant that generically depends on some Information Bearing

- PrintedObject is an object that is and output of some Act of Additive Manufacturing.

- FinishedObject is an object that is an output of some Act of Post-Processing. 


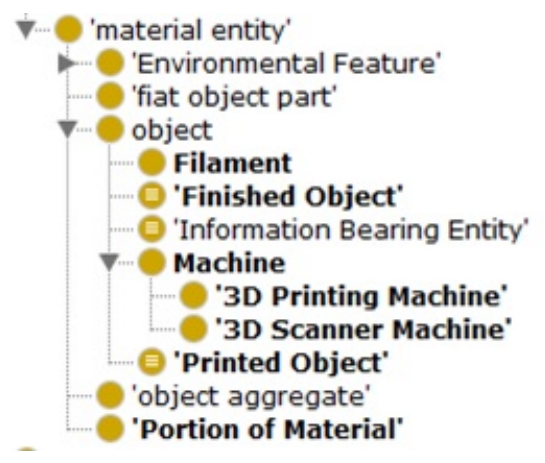

Figure 8: Material Entity classes in AMO.

Entity and stands in the relation of aboutness to some entity ${ }^{4}$ '. The CCO also provides three sub-relations of ' $i s_{-} a b o u t$ ', including: describing, prescribing, and designating.

The AMO makes use of these distinctions provided in the CCO to provide a series of classes of information that either prescribe, designate, or describe various AMO processes and resources. These are displayed in Figure 9 and newly added classes appear in bold. CADSoftwareProgram, CADModel, STLDataFile, and TechnicalDrawing are InformationContentEntity in AMO. Thus, all are generically dependent continuants in BFO. A CADModel, for example, contains 420 information pertaining to the qualities that must inhere in a solid model of the sort that is represented in an ArtifactModel. An ArtifactModel in the CCO is a subclass of ArtifactDesign. Both classes are defined in the $\mathrm{CCO}^{5}$ as follows:

- ArtifactDesign is a Directive Information Content Entity that is a specification of an object, manifested by an agent, intended to accomplish goals, in a particular environment, using a set of primitive components, satisfying a set of requirements, subject to constraints.

\footnotetext{
${ }^{4}$ See https://github.com/CommonCoreOntology/CommonCoreOntologies/blob/master/ AllCore0ntology.ttl

`See https://github.com/CommonCoreOntology/CommonCoreOntologies/blob/master/ AllCoreOntology.ttl
} 
- ArtifactModel is an Artifact Design that prescribes a common set of functions and qualities that are to inhere in a set of artifact instances.

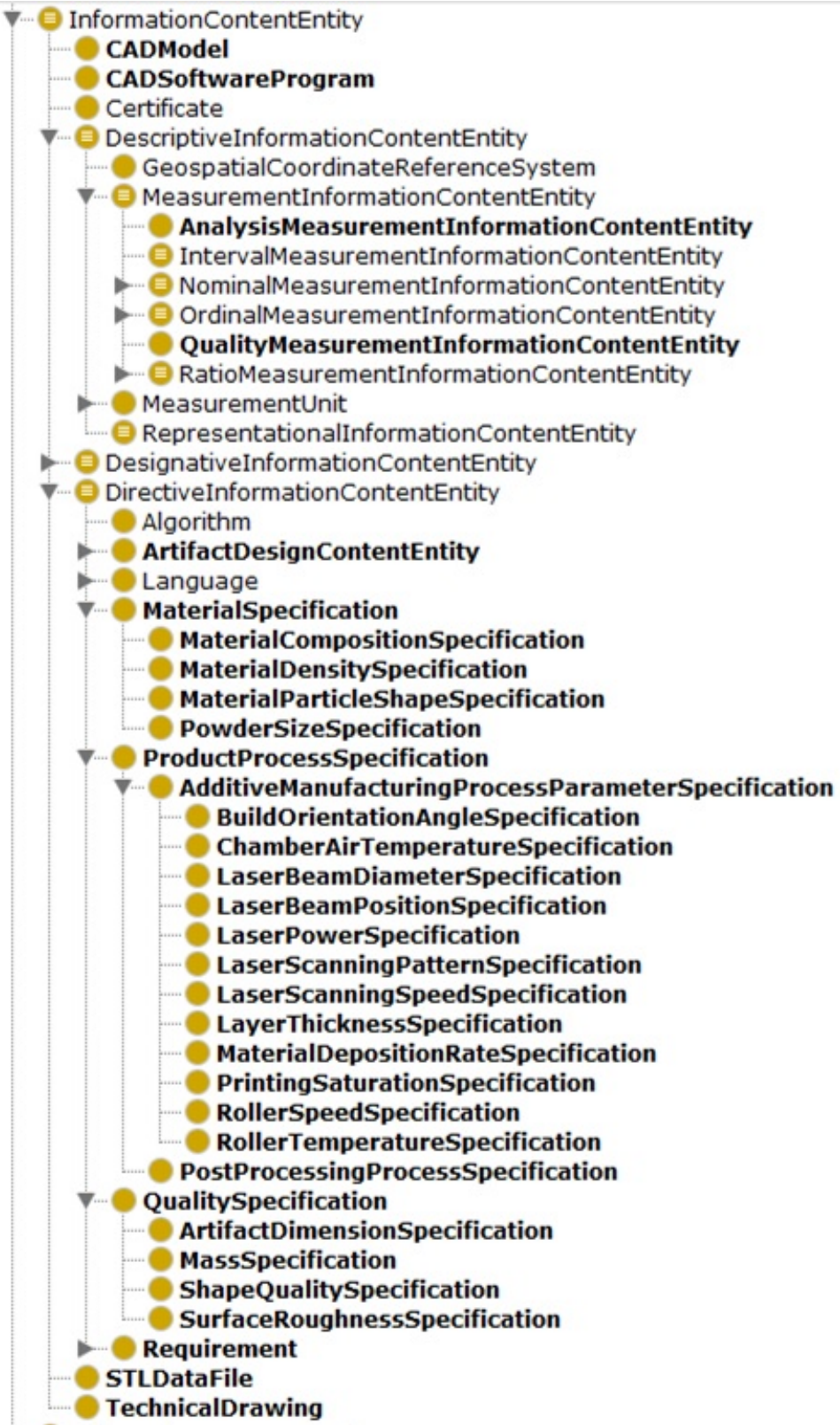

Figure 9: Information Content Entity classes in AMO. 
The CCO class, Directive Information Content Entity is defined as an Infor-

430 are classified under Directive Information Content Entity, as for example where some Additive Manufacturing Process Specification prescribes some Act of Additive Manufacturing.

Requirements, too, are classified under Directive Information Content Entity, 435 inhere in a product. Standards documents such as ISO 3923, the International Standard for Metallic Powders, prescribes the level of quality that a metallic product must have if it is to satisfy the standard.

\subsection{Relations}

Figure 10 provides an overview of the relations used in AMO, in addition to process_part_of discussed in Section 4.1.

Definitions from BFO and the CCO are as follows:

- has_participant is a primitive instance-level relation between a process, a continuant, and a time at which the continuant participates in some way in the process.

- is_input_of is a relation between a continuant and a process in which the continuant participates. The presence of the continuant at the beginning of the process is a necessary condition for the initiation of the process.

- is_output_of is a relation between a continuant and a process in which the 450 continuant participates. The presence of the continuant at the end of the process is a necessary condition for the completion of the process.

- prescribes is for all types $\mathrm{T} 1$ and $\mathrm{T} 2$, if $\mathrm{T} 1$ prescribes $\mathrm{T} 2$, then there is some instance of $\mathrm{T} 1$, $\mathrm{t} 1$, that serves as a rule or guide to some instance of T2, t2. 


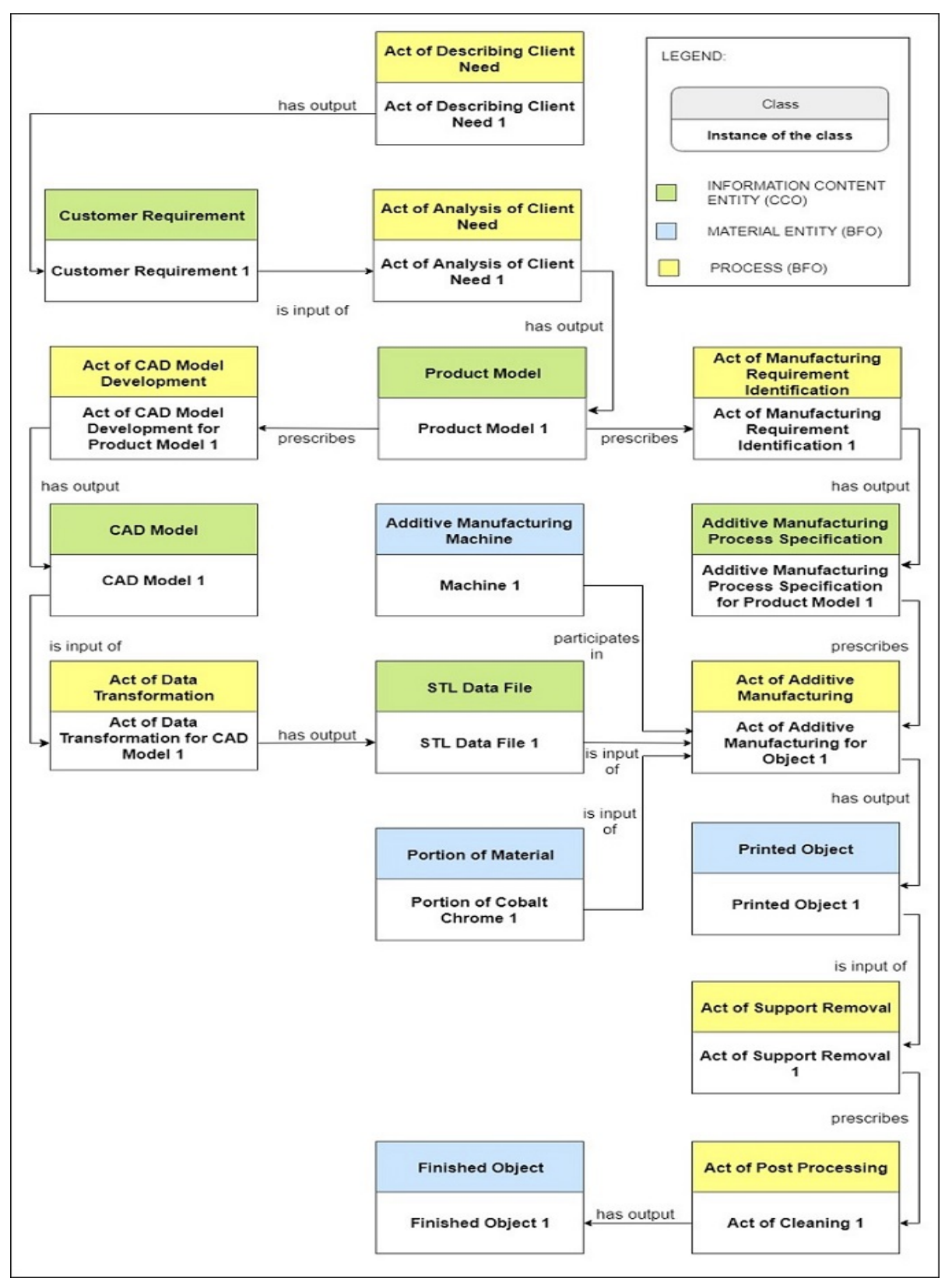

Figure 10: An overview of AMO entities and relations. 


\section{Application of AMO to the Dentistry Product Manufacturing}

AMO was developed to serve as a mid-level ontology that can be re-utilized for multiple different types of additive manufacturing. By providing terms for all processes within the AM organized process flow, we feel that AMO is particularly well suited for PLC applications. Besides, to ensure generality of AMO, the properties interrelating objects of different ontologies are only defined directly in the AMO at the instance level. To show the utility of the developed AMO for developing application-specific ontologies, a case study applying AMO to dentistry manufacturing application termed Additive Manufacturing for Dental Product Ontology (AMDO) is discussed next.

\subsection{Additive Manufacturing (AM) in Dentistry}

AM has established itself in the dentistry field as a promising alternative to the conventional manufacturing processes. AM has the advantage of yielding accurate one-off fabrication of complex structures in a variety of materials having properties highly desirable for both dentistry and surgery [44. AM even becoming a feature of many dental surgeries [45, where it allows direct fabrication of dental prostheses such as crowns and bridges.

As a case study, an application ontology extending AMO with new classes related to the dentistry product manufacturing field has been developed. This application ontology is titled the Additive Manufacturing for Dental Product 475 Ontology (AMDO) and is depicted in Figure 11 (newly added classes are highlighted in bold). As can be seen from the figure, the newly added classes are the extension of the existing classes from the imported ontologies. This shows the re-usability of AMO in the developing the application ontology. No new object properties are needed to be created as well. 


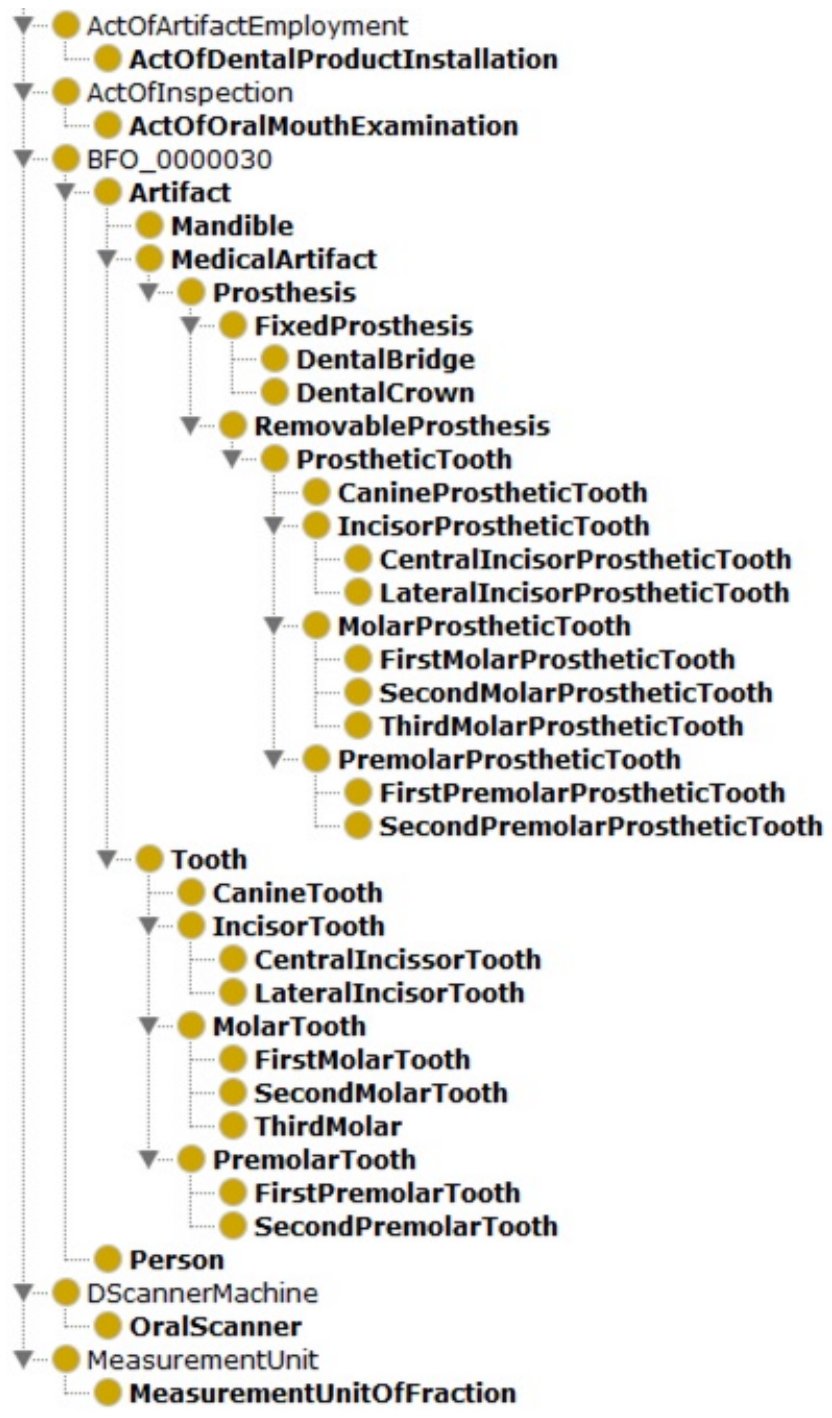

Figure 11: Active classes in AMDO.

\subsection{Case Demonstration}

This section demonstrate an example of the practical uses of the AMDO guided by the work outlined in Khalil et al. 46]. In their work, they evaluate dimensional differences between natural teeth and the printed models using three different AM processes. The process starts with the scanning of three premolars 
to yield the three-dimensional data needed for the dental model. Table 2 shows the specifications of the four 3D printers used in the study Khalil et al. 46].

\begin{tabular}{|c|c|c|c|c|}
\hline Machine & SLA & Objet Eden 250 & Objet Connex 350 & UP Plus 2 \\
\hline Printing type & SLA & Polyjet & Polyjet & FDM \\
\hline Layer thickness $(\mu \mathrm{m})$ & 50 & 16 & 16 & 150 \\
\hline Material used & Resin & Resin & Resin & ABS \\
\hline
\end{tabular}

24 printed premolar tooth were produced in total and the volume of each replicas are measured and compared against the original premolars. Table 3 shows the overview of the volume measurements of each printed premolar tooth with the percentage of volume difference with the original premolars.

Table 3: Volume and Percentage of Volume Differences Data

\begin{tabular}{cccccc}
\hline Group & Tooth No. & UP Plus 2 & Objet Connex 350 & Objet Eden 250 & SLA \\
\hline M1 & 34 & $509.2(4.2 \%)$ & $502.1(2.7 \%)$ & $482.6(-1.3 \%)$ & $484.2(-0.9 \%)$ \\
M1 & 44 & $616.7(18.5 \%)$ & $521.2(0.1 \%)$ & $516.2(-0.9 \%)$ & $519.3(-0.3 \%)$ \\
M1 & 54 & $440.5(-1.6 \%)$ & $438.8(-2.0 \%)$ & $446.2(-0.3 \%)$ & $448.0(0.1 \%)$ \\
M2 & 34 & $376.5(-4.4 \%)$ & $421.6(7.0 \%)$ & $398.2(1.1 \%)$ & $388.9(-1.3 \%)$ \\
M2 & 44 & $340.2(-6.8 \%)$ & $351.0(-3.8 \%)$ & $361.0(\%-1.1 \%)$ & $362.1(-0.8 \%)$ \\
M2 & 54 & $416.4(-12 \%)$ & $464.2(-1.9 \%)$ & $482.6(\%-0.9 \%)$ & $471.1(-0.4 \%)$ \\
\hline
\end{tabular}

For the testing purposes of AMDO, we populated all data from Table 2 and Table 3 as instances for the classes in AMDO. Following are the three queries that we have made for the AMDO to provide the inferred information:

1. What are the 3D Printers used in the study?

2. What type of materials used for each machine and what are the layer thickness specification in fabricating the printed tooth?

3. What are volume differences of the printed tooth with the uses of different $3 \mathrm{D}$ printers?

The queries have been created using an Resource Description Framework (RDF) query language, SPARQL that is a plugin to Protege. SPARQL is a semantic query language that is able to retrieve and manipulate data stored in the 
$\mathrm{RDF}$ format where the entities and its relations are expressed in the form of subjectpredicateobject. For each queries, following are the namespace and its binded prefixes that were used to identify the URI of the classes:

rdf: http://www.w3.org/1999/02/22-rdf-syntax-ns\# owl: http://www.w3.org/2002/07/owl\# rdfs: http://www.w3.org/2000/01/rdf-schema\# xsd: http://www.w3.org/2001/XMLSchema\# bfo: http://purl.obolibrary.org/obo/ ros: http://www.obofoundry.org/ro/ro.owl\# ccos: http://www.ontologyrepository.com/CommonCoreOntologies/ plco: http://www.semanticweb.org/no/ontologies/2017/1/PLC-ontology/ mpos: http://www.semanticweb.org/ontologies/ManufacturingProcessOntolog $515 \mathrm{y} /$ dos: http://www.semanticweb.org/no/ontologies/2017/6/DesignOntology/ amos: http://www.semanticweb.org/munira/ontologies/2017/6/AdditiveMan ufacturingOntology\# amdo: http://www.semanticweb.org/munira/ontologies/2018/1/AdditiveMan ufacturingDentalOntology\#

Query 1: Identifying 3D printers used in the study. The SPARQL Query for this question is as follow:

\section{SELECT ?Machine}

525 WHERE \{

?Machine rdf:type amos:3DPrintingMachine

As can be seen from Table 2, there are four 3D printers used in the study. Even though there are only four data, this simple query represents those with large 


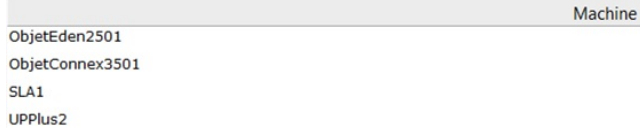

Figure 12: Query 1 Result

Query 2: Identifying type of materials used for each machine and what are the layer thickness specification in fabricating the printed tooth.

${ }_{535}$ To identify the type of materials used for each machine and with the layer thickness specification for the printing process, the relations between the process, machine, materials and also the process specification are defined at the instance level. To increase the selectivity of the inferred information, we limited type of printing process to only to the Vat Photopolymerisation Process. The SPARQL 540 Query for this question is as follow:

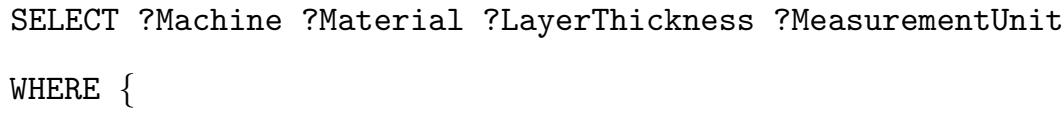




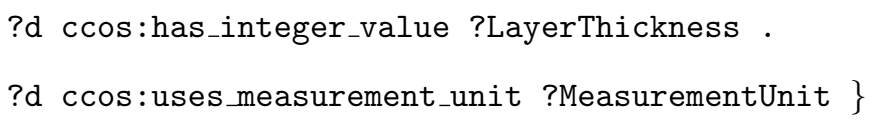

555 This query increases in complexity where there are four classes are involved and it has high selectivity due to the contraint added to one of the class. Figure 13 shows the result of this query.

\begin{tabular}{|c|c|c|c|}
\hline Machine & Material & LayerThickness & MeasurementUnit \\
\hline SLA1 & "Photopolymer Resin"@ & "50"^^<http://www.w3.org/2001/XMLSchema\#integer> & 'Micrometer Measurement Unit' \\
\hline SLA1 & "Photopolymer Resin"@ & "50"^^〈http://www.w3.org/2001/XMLSchema\#integer> & 'Micrometer Measurement Unit' \\
\hline SLA1 & "Photopolymer Resin"@ & "50"^^<http://www.w3.org/2001/XMLSchema\#integer> & 'Micrometer Measurement Unit' \\
\hline SLA1 & "Photopolymer Resin"@ & "50"^^<http://www.w3.org/2001/XMLSchema\# integer> & 'Micrometer Measurement Unit' \\
\hline SLA1 & "Photopolymer Resin"@ & "50"^^〈http://www.w3.org/2001/XMLSchema\#integer> & 'Micrometer Measurement Unit' \\
\hline
\end{tabular}

Figure 13: Query 2 Result

Query 3: Identifying volume differences of the printed tooth with the uses of different $3 \mathrm{D}$ printers?

To identify the volume differences of the printed tooth with the uses of different $3 \mathrm{D}$ printers, the relations between the process, machine, and also the volume measurements with the volume difference analysis data are defined at the instance level. The illustration of the relations between instances of each class is shown in Figure 14.

To increase the selectivity of the inferred information, we limited type of printing process to only to the Vat Photopolymerisation Process. The SPARQL Query for this question is as follow:

SELECT?Machine?ProstheticTooth?ProstheticToothVolume?VolumeDifference

Percentage

WHERE \{

?a rdf:type amos:ActOfMaterialJetting . 


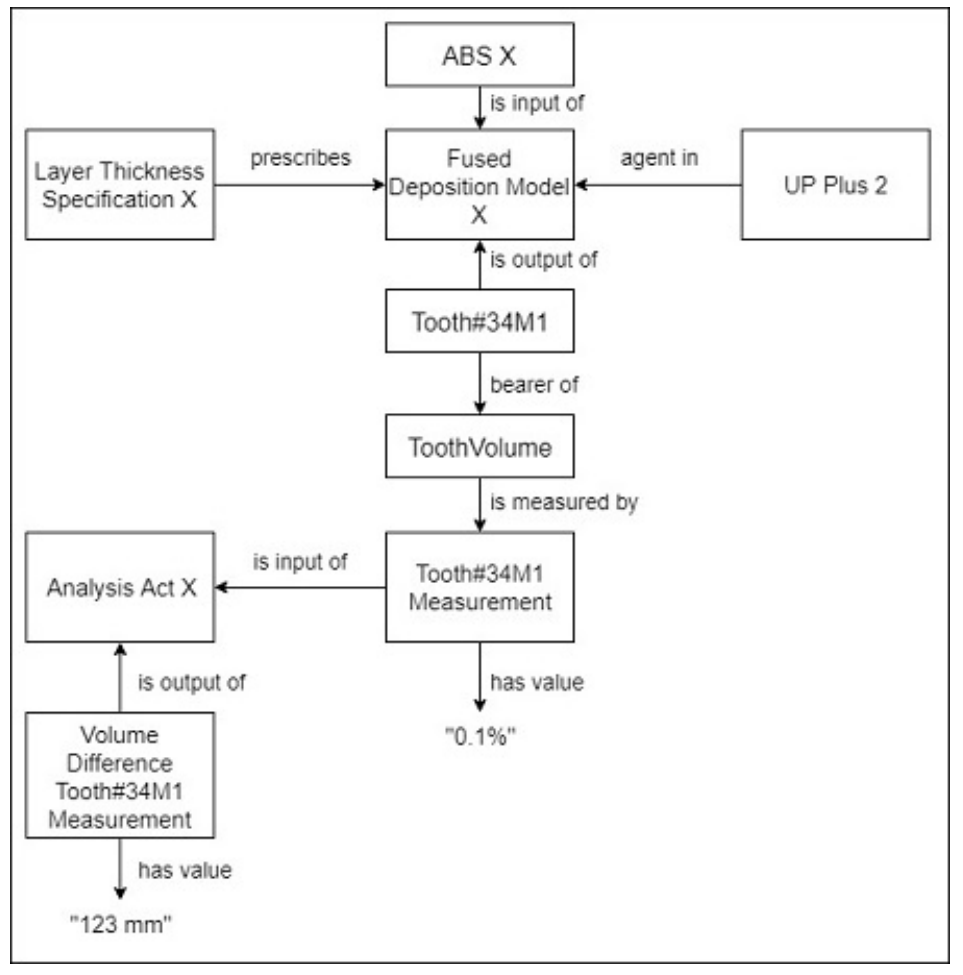

Figure 14: Instances in AMDO

?Machine rdf:type amos:3DPrintingMachine .

?ProstheticTooth ccos:is_output_of ?a .

?ProstheticTooth rdf:type amdo:PremolarProstheticTooth .

?ProstheticTooth ccos:bearer_of ?d.

?d rdf:type amos:VolumeCapacity .

?d ccos:is_measured_by ?e .

?e rdf:type amos:QualityMeasurementInformationContentEntity .

?e ccos:inheres_in ?f.

?f rdf:type ccos:InformationBearingEntity .

?f ccos:has_decimal_value ?ProstheticToothVolume .

?e ccos:is_input_of ?g .

?g rdf:type amos:ActOfAnalysisOfInspectionData . 


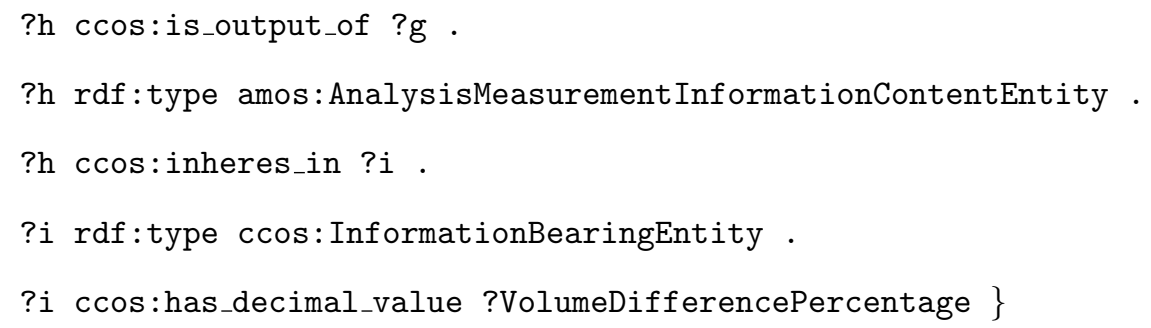

Figure 15 shows the result of this query.

\begin{tabular}{|c|c|c|c|}
\hline Machine & ProstheticTooth & ProstheticToothVolume & VolumeDifferencePercentage \\
\hline ObjetEden 2501 & 'Premolar Tooth 34 Mandible1 (OE250)' & "482.6"^^<http://wWw.w3.org/2001/XML: & :"-1.3"^^<http://www.w3.org/2001/XMLSC \\
\hline ObjetEden 2501 & 'Premolar Tooth 44 Mandible1 (OE250)' & "516.2"^^<http://www.w3.org/2001/XML: & :"-0.9"^^<http://www.w3.org/2001/XMLSc \\
\hline ObjetEden 2501 & 'Premolar Tooth 45 Mandible1 (OE250)' & "446.2"^^<http://www.w3.org/2001/XML: & : -0.3"^^<http://www.w3.org/2001/XMLSc \\
\hline ObjetEden 2501 & 'Premolar Tooth 34 Mandible2 (OE250)' & "398.2"^^<http://www.w3.org/2001/XML: & 1.1"^^^<http://www.w3.org/2001/XMLSCt \\
\hline ObjetEden 2501 & 'Premolar Tooth 44 Mandible2 (OE250)' & "361.0"^^<http://www.w3.org/2001/XML: & " "-1.1"^^<http://www.w3.org/2001/XMLSC \\
\hline ObjetEden 2501 & 'Premolar Tooth 45 Mandible2 (OE250)' & "469.1"^^<http://www.w3.org/2001/XML: & "-0.9"^^<http://www.w3.org/2001/XMLSC \\
\hline ObjetConnex3501 & 'Premolar Tooth 34 Mandible1 (OC350)' & "502.1"^^<http://www.w3.org/2001/XML: & "2.7"^^<http://www.w3.org/2001/XMLSct \\
\hline ObjetConnex3501 & 'Premolar Tooth 44 Mandible1 (OC350)' & "521.2"^^<http://www.w3.org/2001/XML: & "0.1"^^<http://www.w3.org/2001/XMLScl \\
\hline ObjetConnex3501 & 'Premolar Tooth 45 Mandible1 (OC 350 )' & "438.8"^^<http://www.w3.org/2001/XML: & :-2.0"^^<http://www.w3.org/2001/XMLSc \\
\hline ObjetConnex3501 & 'Premolar Tooth 34 Mandible2 (OC 350$)$ ' & "421.6"^^<http://www.w3.org/2001/XML: & : 7.0"^^<http://www.w3.org/2001/XMLScl \\
\hline ObjetConnex3501 & 'Premolar Tooth 44 Mandible2 (OC350)' & "351.0"^^<http://www.w3.org/2001/XML: & :"-3.8"^^<http://www.w3.org/2001/XMLSc \\
\hline ObjetConnex3501 & 'Premolar Tooth 45 Mandible2 (OC 350 )' & "464.2"^^<http://www.w3.org/2001/XML: & :"-1.9"^^<http://www.w3.org/2001/XMLSc \\
\hline
\end{tabular}

Figure 15: Query 3 Result

The answers to the example of queries show the ability of the ontology to retrieve information that matched to the queries even though the classes in AMDO are imported from different ontologies. This is because, AMDO is an extension of AMO and AMO is an extension of CHAMP which are extended from the $\mathrm{CCO}$ and $\mathrm{BFO}$. We use the import process in developing the ontology to maintain the URI of the classes so that the naming of the class, the class structure, the class definition and the class relations will be standardized in all related ontologies. This will ensure re-usability of the ontology. Even though, we have not tested the interoperability of the ontology yet, but aiming for the re-usability of the ontology is a starting point in achieving interoperability of the ontology.

Nevertheless, due to the import structure of the AMDO with the AMO,CHAMP, $\mathrm{CCO}$ and $\mathrm{BFO}$, we will have $\mathrm{CCO}$ terms available in AMDO. The $\mathrm{CCO}$ terms 
may then extend the reach of AMDO to cope with corresponding data concerning persons and organizations, roles of persons (for instance dental technician, patient), measurement units, and cost factors. Thus, the functionality of AMDO can be extended to cope with a digital record or a patients history record in such a way as to document the process of maintenance of the dental crown, comparing susceptible of wear of the crown and of associated dental disorders in different patients, perhaps incorporating also terms from the OBOFoundry ${ }^{6}$.

\section{Conclusion and Future Work}

The AMO was developed within the context of a more general treatment of the PLC. It will be helpful to users who employ AM in their work, and who face the challenge of data integration faced by most modern industries today. It can assist the designer in designing a new product, by enabling access to bodies of data across the entire dentistry product manufacturing domain, for example relating to materials used, patient experiences, maintenance costs, and so forth. The framework is also sufficiently general that it may accommodate the generation of more fine-grained application ontologies in other areas where AM technology is applied.

As the manufacturing industry is evolving rapidly and becoming more competitive, quality and cost are major factors that need to be focused on by the manufacturers. These factors are affected closely by the process and the material. We concentrated here primarily on the process aspect in the AM process but in the next stage, we will work on integrating the AMO with the ontology that 630 represents the types of material used in AM and their associated attributes. This will build on work on the ontology of material that is part of the CHAMP ontologies, where each ontology in CHAMP constitutes a mid-level ontology that

\footnotetext{
${ }^{6}$ See http://www.obofoundry.org/ontology/ohd.html
} 
imports the whole of the $\mathrm{CCO}$, as well as BFO.

\section{References}

[1] X. Chang, R. Rai, J. Terpenny, Development and utilization of ontologies in design for manufacturing, Journal of Mechanical Design 132 (2010) 021009.

[2] A. Gunasekaran, E. Ngai, Knowledge management in 21st century manufacturing, International Journal of Production Research 45 (2007) 2391-2418.

[3] R. Young, A. G. Gunendran, A.-F. Cutting-Decelle, M. Gruninger, Manufacturing knowledge sharing in plm: a progression towards the use of heavy weight ontologies, International Journal of Production Research 45 (2007) 1505-1519.

[4] T. Economist, Knowledge management in manufacturing, 2007. URL:

WWw.01net.it/wp-content/uploads/sites/14/2014/10/siemens_ugs_ Knowledge_management.pdf

[5] S. Lemaignan, A. Siadat, J.-Y. Dantan, A. Semenenko, Mason: A proposal for an ontology of manufacturing domain, in: Distributed Intelligent Systems: Collective Intelligence and Its Applications, 2006. DIS 2006. IEEE Workshop on, IEEE, 2006, pp. 195-200.

[6] C. Welty, D. L. McGuinness, M. K. Smith, Owl web ontology language guide, W3C recommendation, W3C (February 2004) http://www. w3. org/TR/2004/REC-owl-guide-20040210 (2004).

[7] B. Motik, P. F. Patel-Schneider, B. Parsia, C. Bock, A. Fokoue, P. Haase, R. Hoekstra, I. Horrocks, A. Ruttenberg, U. Sattler, Owl 2 web ontology language: Structural specification and functional-style syntax, W3C recommendation 27 (2009) 159. 
[8] T. Kjellberg, A. von Euler-Chelpin, M. Hedlind, M. Lundgren, G. Sivard, D. Chen, The machine tool modela core part of the digital factory, CIRP annals 58 (2009) 425-428.

[9] M. A. Musen, Upper Level Ontology. In: Dubitzky, W., Wolkenhauer, O., Yokota, H., Cho, K-H.(eds)Encyclopedia of systems biology, Springer Publishing Company, Incorporated, 2013.

[10] Ramos, 2011. URL: https://downloads.sourceforge.net/project/ ontomanufacture/mop/Machine_Ontology.owl.

[11] L. Ramos, R. Gil, D. Anastasiou, M. J. Martin-Bautista, Towards a machine of a process (mop) ontology to facilitate e-commerce of industrial machinery, Computers in Industry 65 (2014) 108-115.

[12] L. Mazzola, P. Kapahnke, M. Vujic, M. Klusch, Cdm-core: A manufacturing domain ontology in owl2 for production and maintenance, in: KEOD, ????, $670 \quad$ pp. $136-143$.

[13] F. Ameri, C. Urbanovsky, C. McArthur, A systematic approach to developing ontologies for manufacturing service modeling, in: Proc. 7th International Conference on Formal Ontology in Information Systems (FOIS 2012), Graz, Austria, Citeseer, 2012.

675

[14] L. Mesmer, A. Olewnik, Development of a part-focused manufacturing process ontology: Exploring use and applications, in: ASME 2015 International Design Engineering Technical Conferences and Computers and Information in Engineering Conference, American Society of Mechanical Engineers, 2015, pp. V01BT02A034-V01BT02A034.

680

[15] B. Smith, 2017. URL: https://emmc.info/wp-content/uploads/2017/ 12/EMMC-IntOp2017-Cambridge_Smith_Buffalo.pdf 
[16] Y.-J. Chen, Y.-M. Chen, H.-C. Chu, Development of a mechanism for ontology-based product lifecycle knowledge integration, Expert Systems with Applications 36 (2009) 2759-2779.

[17] M. B. Raza, R. Harrison, Ontological knowledge based system for product, process and resource relationships in automotive industry, in: Proceedings of the 1st International Workshop on Ontology and Semantic Web for Manufacturing, Heraklion, Crete, Greece, ????, pp. 23-36.

[18] M. Borsato, Bridging the gap between product lifecycle management and sustainability in manufacturing through ontology building, Computers in Industry 65 (2014) 258-269.

[19] H. Cao, P. Folan, Product life cycle: the evolution of a paradigm and literature review from 1950-2009, Production Planning \& Control 23 (2012) 641-662.

[20] A. Matsokis, D. Kiritsis, An ontology-based approach for product lifecycle management, Computers in industry 61 (2010) 787-797.

[21] N. Chungoora, R. Young, G. Gunendran, Z. Usman, N. Anjum, C. Palmer, J. Harding, K. Case, A. CuttingDecelle, A model driven ontologybased approach for manufacturing knowledge sharing in plm, Enterprise Interoperability: IESA'12 Proceedings (2012) 197-204.

[22] Z. Usman, R. I. M. Young, N. Chungoora, C. Palmer, K. Case, J. Harding, A manufacturing core concepts ontology for product lifecycle interoperability, in: International IFIP Working Conference on Enterprise Interoperability, Springer, ????, pp. 5-18.

[23] E. Urwin, Deliverable D3.2: Initial formal ontologies, Report, 
$2 \% 20$ Initial $\% 20$ Formal $\% 200$ ntologies.pdf

[24] E. Urwin, C. Palmer, B. Young, E. Palacios, J. M. Pinazo, A. Font, R. Almarcha, Deliverable D3.5: Product-service-production ontologies report, Report, 2016. URL: http://www.flexinet-fof.eu/Documents/FLEXINET\% 20D3.5\%20Product-Service-Production\%200ntologies.pdf.

[25] A. Standard, Iso/astm 52900: 2015 additive manufacturing-general principles-terminology, ASTM F2792-10e1 (2012).

[26] D. Eddy, S. Krishnamurty, I. Grosse, M. Perham, J. Wileden, F. Ameri,

[27] M. Dinar, D. W. Rosen, A design for additive manufacturing ontology,

[28] NIST, Nist's owl ontology for additive manufacturing, 2016. URL: https: //github.com/iassouroko/AMontology.

[29] B.-M. Roh, Ontology-Based Laser Metamodels and Visualization for MetalBased Additive Manufacturing, Thesis, 2017.

[30] J. S. Liang, An ontology-oriented knowledge methodology for process planning in additive layer manufacturing, Robotics and Computer-Integrated Manufacturing 53 (2018) 28-44.

[31] T. J. Hagedorn, S. Krishnamurty, I. R. Grosse, A knowledge-based method 
ontologies, Journal of Computing and Information Science in Engineering 18 (2018) 021009.

[32] N. Natalya F, M. Deborah L, Ontology development 101: A guide to creating your first ontology, 2001.

735
URL: https://github.com/CommonCoreOntology/ CommonCoreOntologies/blob/master/Best\%20Practices\%20of\% 200ntology\%20Development.pdf.

[34] R. Arp, B. Smith, A. D. Spear, Building ontologies with basic formal ontology, Mit Press, 2015.

[35] V. Mascardi, V. Cord, P. Rosso, A comparison of upper ontologies, in: Woa, volume 2007, ????, pp. 55-64.

[36] S. Borgo, P. Leito, The role of foundational ontologies in manufacturing domain applications, in: OTM Confederated International Conferences" On the Move to Meaningful Internet Systems", Springer, 2004, pp. 670-688.

[37] CUBRC, Common Core Ontologies for Data Integra-

n tion, 2017. URL: https://www.cubrc.org/index.php/ data-science-and-information-fusion/ontology.

[38] F. Furini, R. Rai, B. Smith, G. Colombo, V. Krovi, Development of a manufacturing ontology for functionally graded materials, in: ASME 2016 International Design Engineering Technical Conferences and Computers and Information in Engineering Conference, American Society of Mechanical Engineers, 2016, pp. V01BT02A030-V01BT02A030.

[39] B. Smith, Basic Formal Ontology 2.0 Specification and User's Guide, 2015. URL: https://github.com/BFO-ontology/BFO/raw/master/docs/ bfo2-reference/BF02-Reference.pdf. 
[40] W. Ceusters, B. Smith, Aboutness: Towards foundations for the information artifact ontology, in: Proceedings of the Sixth International Conference on Biomedical Ontology (ICBO), volume 1515, CEUR, 2015, pp. 1-5.

[41] CUBRC, An Overview of the Common Core Ontologies, 2017. URL: https://github.com/CommonCoreOntology/CommonCoreOntologies/

blob/master/An\%200verview $\% 20$ of $\% 20$ the $\% 20$ Common $\% 20$ Core $\%$ 200ntologies\%201.0.pdf.

[42] L. Yang, K. Hsu, B. Baughman, D. Godfrey, F. Medina, M. Menon, S. Wiener, Additive Manufacturing of Metals: The Technology, Materials, Design and Production, Springer, 2017.

[43] B. Smith, On classifying material entities in basic formal ontology, in: Interdisciplinary Ontology: Proceedings of the Third Interdisciplinary Ontology Meeting, Keio University Press, 2012, pp. 1-13.

[44] A. Barazanchi, K. C. Li, B. AlAmleh, K. Lyons, J. N. Waddell, Additive technology: update on current materials and applications in dentistry, Journal of Prosthodontics 26 (2017) 156-163.

[45] A. Dawood, B. M. Marti, V. Sauret-Jackson, A. Darwood, 3d printing in dentistry, British dental journal 219 (2015) 521.

[46] W. Khalil, M. EzEldeen, E. Van De Casteele, E. Shaheen, Y. Sun, M. Shahbazian, R. Olszewski, C. Politis, R. Jacobs, Validation of cone beam computed tomographybased tooth printing using different three-dimensional printing technologies, Oral surgery, oral medicine, oral pathology and oral radiology 121 (2016) 307-315. 